\title{
What's our trach record-Impact of Sars-Cov2 on tracheostomy practice
}

\author{
Holly Jones ${ }^{1,2,3 *}$, Justin Hintze ${ }^{1,2,3}$, Conall Fitzgerald ${ }^{1,2,3}$, John Kinsella ${ }^{1,2,3}$, Paul Lennon ${ }^{1,2,3}$ and Conrad Timon ${ }^{1,2,3}$ \\ ${ }^{1}$ Department of Otolaryngology - Head and Neck Surgery, St. James's Hospital, Dublin, Ireland \\ ${ }^{2}$ Trinity College Dublin, University of Dublin, Ireland \\ ${ }^{3}$ Royal College of Surgeons in Ireland
}

\begin{abstract}
Background: Tracheostomies are commonly performed procedures. Risk of transmission of Covid-19 is considered high during formation of surgical tracheostomy and in the post-operative period, creating a significant clinical challenge.

Aims/objectives: To identify areas of improvement for patients with tracheostomies and assess the impact of the Covid-19 pandemic on the rate of tracheostomy insertion.

Materials and methods: Patients who underwent a tracheostomy in 2020 in a single tertiary referral centre were prospectively identified.

Results: During the five-month period 61 tracheostomies were performed. The most common indication was prolonged mechanical ventilation (68.8\% $\mathrm{n}=42$ ), followed by prophylactic airway management $(27.8 \% \mathrm{n}=17)$ and emergency airway management $(3.2 \% \mathrm{n}=2)$. Four tracheostomies were performed in patients who tested positive for Covid-19, all were percutaneous. Three procedure-related complications occurred, all involving minor bleeding. Following tracheostomy $27.8 \%$ of patients were cared for only in ICU, 19.6\% were cared for on a ward familiar with tracheostomy care and 39.3\% were cared for on a general ward. This data was not available for 13\%. Over the same 5-month period in 2019 a total of 79 tracheostomies were performed, 29\% percutaneous and $71 \%$ surgical.
\end{abstract}

Conclusions and significance: Surgical tracheostomy is an essential procedure in the management of a variety of patients.

\section{Background}

Tracheostomy is a commonly surgical procedure performed with numerous indications. These include prolonged mechanical ventilation, prophylactic airway management and emergency airway obstruction. In the critical care setting, tracheostomy provides advantages over prolonged endotracheal intubation, including reducing dead space ventilation, decreased length of intensive care unit (ICU) stay and improved secretion clearance [1,2]. At least $10 \%$ of patients requiring 3 or more days of mechanical ventilation will eventually receive a tracheostomy [3].

Tracheostomy insertion is completed via open surgical or percutaneous approach. Major tracheostomy complications are rare but when they occur can be life threatening. These include obstruction, accidental dislodgement, paratracheal 'false passage' insertion, haemorrhage, tracheal or oesophageal injury and pneumothorax [4]. With rising number of tracheostomies being performed, these patients are typically cared for in multiple locations throughout the hospital, including general wards [5].

\section{Materials and methods}

All patients undergoing tracheostomy in a single tertiary referral centre for head and neck cancer, and the largest ICU in the country were prospectively identified over a 5-month time period, commencing in January 2020. Data was extracted from Power chart electronic patient record and IntelliSpace Critical Care and Anaesthesia (ICCA) information system. Data collected were patient demographics, indication for tracheostomy, type of tracheostomy (percutaneous or surgical), procedure-related complications, tracheostomy changes, days to decannulation and location in the hospital the patient was cared for.

To determine the impact of the SARS-Cov2 pandemic on tracheostomy practice, the data was compared to the numbers of tracheostomies performed at the same time-period 1 year prior. This research was approved by the institutionary review board for Tallaght University Hospital/St. James's Hospital research ethics committee.

\section{Results}

Sixty-one patients underwent tracheostomy during the five-month period in $2020,41 \%$ female and $59 \%$ male. The mean age at time of insertion was 61.9 years. There were 30 percutaneous and 31 surgical tracheostomies performed. All percutaneous tracheostomies $(49 \%$, $\mathrm{n}=30$ ) performed were in patients requiring prolonged mechanical ventilation and were performed in ICU.

Surgical tracheostomies were performed in an operating theatre by the otolaryngology/head and neck surgery service. The most

${ }^{\star}$ Correspondence to: Holly Jones, Department of Otolaryngology-Head and Neck Surgery, St. James's Hospital, James's Street, Dublin, Ireland, Tel: 014103000, E-mail: HollyJones@rcsi.ie

Key words: MicroRNA, miR-26b, TSCC, Cal27, therapeutic target

Received: January 04, 2021; Accepted: January 18, 2021; Published: January 21, 2021 
common indication for surgical tracheostomy was prophylactic airway management $(61 \%, n=19)$, followed by prolonged mechanical ventilation $(39 \%, \mathrm{n}=12)$. In the latter group, 11 patients were unsuitable for percutaneous procedure due to unfavourable anatomy (e.g., high body mass index or short neck) and one patient was coagulopathic. In patients who were SARS-Cov2 negative where tracheostomy was performed for prolonged mechanical ventilation, the mean number of days of invasive ventilation prior to tube insertion was 12.8 days. In the four patients who were positive for SARS-Cov2, the mean number of days of ventilation prior to tracheostomy was 20.7 days.

All patients were initially cared for in the ICU post procedure. Three procedure-related complications were recorded. All complications occurred during percutaneous tracheostomy insertion and involved minor bleeding controlled by local measures, for example silver nitrate cautery or direct local pressure.

Overall, 26 patients underwent a tracheostomy tube change prior to decannulation. In the prolonged ventilation group, 14 patients underwent a tracheostomy tube change, and this occurred on average at 28 days post-insertion. Twelve patients underwent a tube change in the prophylactic airway group and on average this occurred at day 10.

The average number of days to decannulation was 32.3 in patients requiring prolonged mechanical ventilation and 15.6 in prophylactic airway management. Fourteen patients died with tracheostomy in situ. Seven patients were discharged or transferred from the hospital with tracheostomy in situ.

In the prolonged ventilation group, $44 \%(n=17)$ of patients were cared for in the ICU alone. Of the patients who were discharged from ICU, only $8 \%(n=3)$ went to the head and neck ward, while $36 \%$ $(\mathrm{n}=14)$ were cared for on general wards. This data was not available for five patients. In the prophylactic airway management group, $47 \%$ of patients were discharged from ICU to the head and neck ward and $53 \%$ were cared for on a general ward.

Four tracheostomies were performed in patients who tested positive for SARS-Cov2, all were percutaneous. These patients were ventilated on average for 21 days prior to the procedure. Nine patients developed SARS-Cov2 after tracheostomy insertion, with six of these infections occurring post decannulation. The mean number of days after decannulation to developing SARS-Cov2 was 20.5 days.

Retrospective review of tracheostomy practice in 2019 showed a total of 79 tracheostomies over the same 5-month period, $29 \%$ percutaneous and $71 \%$ surgical. While the mean number of tracheostomies in January, February and March were roughly equal between 2019 and 2020, April and May showed a marked reduction in the number of tracheostomies performed in 2020, with a shift to predominantly percutaneous tracheostomies. While in 2019 a total of 30 tracheostomies were performed during those 2 months, this dropped to only 13 in 2020, only 1 of which was performed surgically.

\section{Discussion}

Tracheostomies are used primarily in head and neck surgery and the ICU setting. They are most commonly performed to facilitate weaning from mechanical ventilation in critically ill patients or as prophylactic airway management for large head and neck resection. These patients have complex needs that require specialist multi-disciplinary input and patient education.

Despite known advantages of tracheostomy in the critical care setting, optimal timing remains controversial. Whether tracheostomy is performed early, within four days, or late, after ten days, in patients requiring prolonged mechanical ventilation remains unresolved in the literature [6]. Although no optimal timing has been identified, tracheostomy is typically not performed before ten days of mechanical ventilation. Early tracheostomy may lead to unnecessary surgery and prolonged mechanical ventilation in those who may otherwise be extubated [7]. It is widely accepted that patients should not be ventilated by endotracheal tube for longer than three weeks unless they are unstable or unlikely to benefit from tracheostomy $[8,9]$. Four percutaneous tracheostomies were performed in patients with SARSCov2 to facilitate ventilation weaning. The mean number of days of mechanical ventilation prior to the procedure in these patients was considerably longer than those without SARS-Cov2, 20.7 vs 12.8 days. The procedure should be delayed in these patients, until at least day fourteen of ventilation, to allow a reduction in viral load and when there is an increased likelihood of recovery, where weaning ventilation is the main goal of care $[10,11]$. Thus, the decision to perform and timing of a tracheostomy in the critical care setting is a collaborative one and is guided by the overall clinical picture.

Complications of tracheostomy placement are infrequent but can be life threatening. The incidence of major or minor bleeding during tube insertion has been reported as 5.7\% [12]. Although major bleeding is rare, minor bleeding can lead to life threatening airway obstruction. In our study there were three procedure related complications involving minor bleeding, all of which occurred during percutaneous tracheostomy insertion. Oliver et al demonstrated significantly fewer minor early complications with surgical tracheostomy compared to percutaneous placement in a meta-analysis but comparable longterm complications [13]. The percutaneous method is widely accepted as safe and cost effective. It can be done at the bedside and does not require transport of a critically ill patient to theatre.

All tube changes have the potential to be high risk and should be performed in a planned manner where emergency intubation equipment is available. Surgical tracheostomy involves open dissection down to the trachea and secures the newly established airway immediately. In contrast the percutaneous method involves a small skin incision, insertion of a guidewire followed by sequential dilation and insertion of the cannula. In the case of a dislodged tube, it is reasonable to assume that it may be more difficult to recannulate patients who have had a percutaneous tracheostomy. However, the length of time a stoma will remain patent varies from patient to patient but maturation of the tract is generally completed by the seventh post-operative day. There is conflicting evidence on how frequently tracheostomy tubes should be changed and timing is usually driven by local practice. Rather than being performed on a predetermined schedule, tube changes should be performed as the patient's clinical condition dictates. All tubes should be routinely changed every 28-30 days in line with European Union regulations and manufacturer recommendations [14].

In our study patients with tracheostomies were frequently cared for on non-specialised wards. The use of percutaneous tracheostomies has expanded in recent years and this is reflected in our data with close to half the number of tracheostomies being performed percutaneously $49.2 \%$ in 2020 and $29.1 \%$ in 2019. This has led to an overall increase in the number of hospitalised patients with temporary tracheostomies. The team performing the tracheostomy is frequently different to the team caring for the patient and thus the care of the tracheostomy is shared between many healthcare teams from a variety of disciplines [12]. Patients should have locally agreed standardised daily tracheostomy care and a sign above the bedhead that includes key details of the 
tracheostomy as well as an emergency algorithm. Tracheostomy emergency equipment should be readily available on the ward. The UK National Confidential Enquiry into Patient Outcomes and Death (NCEPOD) found that after tracheostomy insertion, patients are most commonly cared for on 2-4 wards during their hospital stay with $7 \%$ of hospitals reporting patients being moved across 10 wards [15]. The National Tracheostomy Safety Project (NTSP) recommends that inpatients with a tracheostomy who do not require a critical care bed be cared for in designated cohort wards, such as the head and neck ward [16]. This allows training and expertise to be concentrated in one clinical area resulting in improved patient safety [17]. Coordinated multidisciplinary care as well as local protocol driven efforts involving all persons involved in tracheostomy care reduces tracheostomy related morbidity and mortality [18].

The SARS-Cov2 pandemic has changed how healthcare is delivered worldwide. Tracheostomies pose a unique challenge in this era. Since the beginning of the SARS-Cov2 crisis there has only been one surgical tracheostomy performed in our institution. The procedure poses a significant risk of transmission to healthcare workers [19]. The reduction in number of tracheostomies performed during the same period in 2019 compared with 2020 is in keeping with international recommendations for performing tracheostomies at this time [11], and also reflects a marked reduction in elective head and neck surgical practice. Due to the pandemic, elective operating has been outsourced to different institutions and there have been no elective tracheostomies performed for prophylactic airway management. From follow up to date, nine patients in this study developed SARS-Cov2 following tracheostomy. This highlights that these patients are particularly vulnerable to viral transmission. Decision making to perform a tracheostomy during this time should be undertaken by a multidisciplinary team with a detailed analysis of risk and benefit.

\section{Conclusions and significance}

A reduction in total number of tracheostomies performed, in particular open surgical tracheostomies, has been observed during the SARS-Cov2 pandemic. Furthermore, these patients are being cared for in multiple locations throughout the hospital which can have significant safety implications for their care. This may have ongoing implications for clinical practice in relation to tracheostomy.

\section{References}

1. Arabi Y, Haddad S, Shirawi N, Al Shimemeri A (2004) Early tracheostomy in intensive care trauma patients improves resource utilization: a cohort study and literature review. Crit Care 8: R347-R352. [Crossref]
2. Hsu CL, Chen KY, Chang CH, Jerng JS, Yu CJ, et al. (2005) Timing of tracheostomy as a determinant of weaning success in critically ill patients. Crit Care 9: R46-R52. [Crossref]

3. Durbin CG Jr (2010) Tracheostomy: why, when, and how? Respirat Care 55: 1056-68.

4. Klotz R, Probst P, Deininger M, Klaiber U, Grummich K, et al. (2018) Percutaneous versus surgical strategy for tracheostomy: a systematic review and meta-analysis of perioperative and postoperative complications. Langenbecks Arch Surg 403: 137-149. [Crossref]

5. Wilkinson KA, Freeth H, Martin IC (2015) Are we 'on the right trach?' The National Confidential Enquiry into Patient Outcome and Death examines tracheostomy care. $J$ Laryngol Otol 129: 212-216. [Crossref]

6. Gomes Silva BN, Andriolo RB, Saconato H, Atallah AN, Valente O (2012) Early versus late tracheostomy for critically ill patients. Cochrane Database Syst Rev 2012: Cd007271. [Crossref]

7. Hosokawa K, Nishimura M, Egi M, Vincent JL (2015) Timing of tracheotomy in ICU patients: a systematic review of randomized controlled trials. Crit Care 19: 424. [Crossref]

8. Stone DJ, Bogdonoff DL (1992) Airway considerations in the management of patients requiring long-term endotracheal intubation. Anesth Analg 74: 276-287.

9. Heffner JE (1993) Timing of tracheotomy in mechanically ventilated patients. AM Rev Respirat Dis 147: 768-771.

10. Centre ICNAR (2020) ICNARC report on COVID-19 in critical care.

11. Givi B, Schiff BA, Chinn SB, Clayburgh D, Iyer NG, et al. (2020) Safety Recommendations for Evaluation and Surgery of the Head and Neck During the COVID-19 Pandemic. JAMA Otolaryngol Head Neck Surg 146: 579-584. [Crossref]

12. Delaney A, Bagshaw SM, Nalos M (2006) Percutaneous dilatational tracheostomy versus surgical tracheostomy in critically ill patients: a systematic review and metaanalysis. Crit Care 10: R55.

13. Oliver ER, Gist A, Gillespie MB (2007) Percutaneous Versus Surgical Tracheotomy: An Updated Meta-Analysis. Laryngoscope 117: 1570-1575.

14. Council directive concerning medical devices, Rule 7. 93 / 42. (1993).

15. Wilkinson K, Freeth H, Kelly K (2015) 'On the Right Trach?' A review of the care received by patients who undergo tracheostomy. Br J Hosp Med (Lond) 76: 163-165. [Crossref]

16. McGrath B (2014) Comprehensive Tracheostomy Care: The National Tracheostomy Safety Project Manual: Wiley.

17. McGrath BA, Thomas AN (2010) Patient safety incidents associated with tracheostomies occurring in hospital wards: a review of reports to the UK National Patient Safety Agency. Postgraduate Med J 86: 522-555.

18. Cetto R, Arora A, Hettige R, Nel M, Benjamin L, et al. (2011) Improving tracheostomy care: a prospective study of the multidisciplinary approach. Clin Otolaryngol 36: 482-488.

19. Michetti CP, Burlew CC, Bulger EM, Davis KA, Spain DA, et al. (2020) Performing tracheostomy during the Covid-19 pandemic: guidance and recommendations from the Critical Care and Acute Care Surgery Committees of the American Association for the Surgery of Trauma. Trauma Surg Acute Care Open 5: e000482. [Crossref]

Copyright: (C2021 Jones H. This is an open-access article distributed under the terms of the Creative Commons Attribution License, which permits unrestricted use, distribution, and reproduction in any medium, provided the original author and source are credited. 